\title{
ESEM Beam Current Measuring Device based on a Planar Schottky Diode
}

\author{
${ }^{1}$ André-Sébastien Aubin, ${ }^{1}$ Dominique Drouin, ${ }^{2}$ Matthew R Phillips \\ ${ }^{1}$ Microelectronics Research Group, Electrical and computer engineering department, Université de \\ Sherbrooke, Sherbrooke (Québec), J1K 2R1, Canada \\ ${ }^{2}$ MAU, University of Technology, Sydney, PO Box 123, Broadway, NSW 2007, Australia
}

Even if Environmental Scanning Electron Microscopes (ESEM) has become a standard instrument, some problems still occur for some type of application such as quantitative microanalysis. In this application the electron beam currents as to be measured in order to determine the appropriate chemical composition of the sample. The existing technology used in standard Scanning Electron Microscope (SEM), can't give an accurate measure because of the ionized gas in the observation chamber. The ions in the chamber create a false current measure in traditional devices, such as Faraday cup. In fact, the resulting current represents more the concentration of ions than the actual electron beam current. To find a solution to this flaw, it is important to develop a device that could measure the electron beam current without being influenced by the ions flood. The making of a planar Schottky junction based device appears a viable solution to this problem.

The accelerated electrons of the beam hit the metal-semiconductor junction and create electron hole pairs within the semiconductor volume. Properly connected, this device induces a current related to the number of electron - hole pair generated within or in the vicinity of the depletion zone. The gain of the device becomes directly related to the current of the electron beam. The current gain created by the ions in the chamber is considerably less important than the current gain of the Schottky diode. Thus, it is possible to obtain accurate value of the beam current even with a gas environment. It is important to choose adequately the metal and semiconductor to form the junction. A platinum silicide appears to be the best choice because of its high extraction function. To increase the electron barrier height and thus the depletion depth, undoped silicon substrate is used. In the back of the diode, for the ground plane, we choose a metallic alloy that will give an ohmic contact, the antimony-gold alloy. This allows the current to flow without restriction. The resulting crosssection of the diode is illustrated in Figure 1.

In the making of this device, we had to be sure that the current measure within the Schottky diode corresponds to the undeflected electrons of the beam to reduce the effect of the skirt. For this reason, we tried to minimize the size of the diode. The result was a $50 \mathrm{~nm}$ large diode with a circular end of $75 \mathrm{~nm}$ in diameter that works as an aim for the beam. To obtain this size, we used electron beam lithography technique with a computer design interface. The tiny size of the diode and the microscope stage lack of precision created difficulties in the alignment of the pad only over the tail end of the diode and not over the complete diode. The solution to this fabrication problem has to give a perfect yield in the alignment of the pad. So we developed a clear and precise procedure to obtain this yield. The cons of this procedure are the addition of a preparation steps to obtain the final result, but this was a limited concession to reach our objective.

To test our solution, three steps were planned. First, a simple current voltage (IV) test to determine the characteristics of the diode. Then, a EBIC test in a standard SEM to allow us to map the gain of the diode, the result of this test are showed in Figure 2. Furthermore, a final test in a ESEM will conclude this experiment and will permit to characterize our device. This result will be presented. 


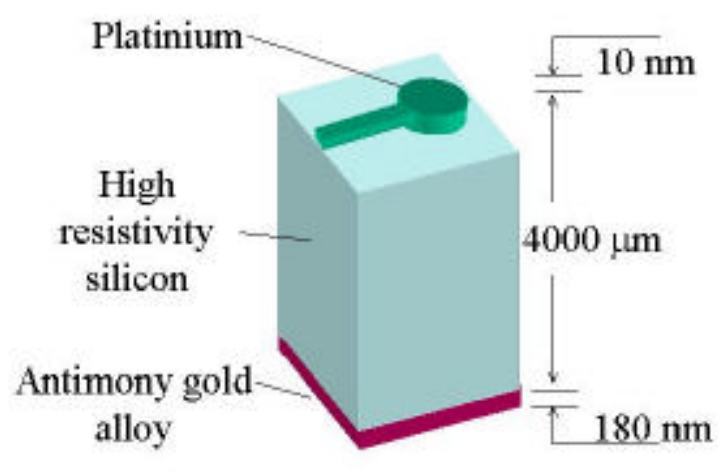

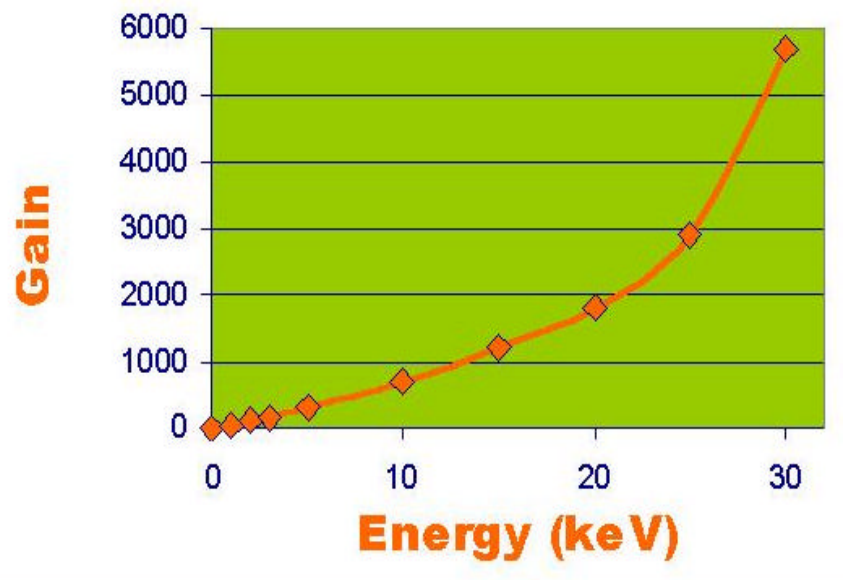

Figure 1 - Planar Schottky diode cross-section Figure 2 - Detector Gain as a function of electron schematic beam energy

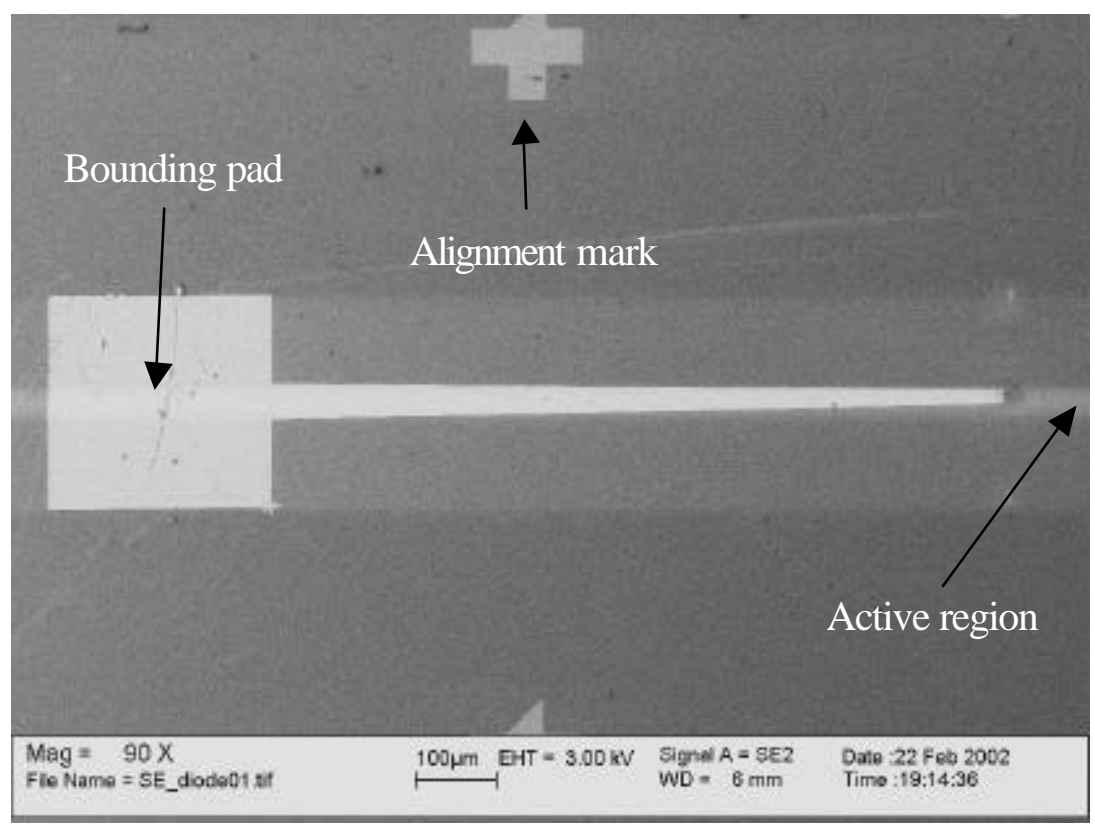

Figure 3 - Micrograph of the planar Schottky Diode top view

\section{References}

R.B. Bolon, Microbeam Analysis, 199 (1991)

D. Drouin et al., Microbeam Analysis 2000, Institute of Physics Conference Series Number 165, 271-272 (2000)

D. Drouin et al., Microscopy and Microanalysis, vol 3, Sup 2, 499-500 (1997).

B.J Griffin, Proceedings 50th EMSA, 1324 (1992)

M.R. Phillps et al., Microscopy and Microanalysis, 5 (S2), 294 (1999)

Acknowledgments

To J.Corbin and P.Magny for their technical assistance. 